\title{
Frege on Referentiality and Julius Caesar in Grundgesetze Section 10
}

\author{
Bruno Bentzen
}

\begin{abstract}
This paper aims to answer the question of whether or not Frege's solution limited to value-ranges and truth-values proposed to resolve the "problem of indeterminacy of reference" in section 10 of Grundgesetze is a violation of his principle of complete determination, which states that a predicate must be defined to apply for all objects in general. Closely related to this doubt is the common allegation that Frege was unable to solve a persistent version of the Caesar problem for value-ranges. It is argued that, in Frege's standards of reducing arithmetic to logic, his solution to the indeterminacy does not give rise to any sort of Caesar problem in the book.
\end{abstract}

\section{The problem of the indeterminacy of reference}

In section 10 of the first volume of Grundgesetze der Arithmetik, Frege (1962) addresses a particular problem about the law he formulated to introduce the notion of value-ranges, namely, the famous Axiom $\mathrm{V}$ : the value-range of the function $f$ is identical to the value-range of the function $g$ just in case $f$ and $g$ have the same values for the same arguments. ${ }^{1}$ In symbols:

$$
\dot{\epsilon}^{\prime} f(\epsilon)=\dot{\alpha} g(\alpha) \leftrightarrow(\forall x)(f(x)=g(x))
$$

${ }^{1}$ All English translations of the first and second volumes of Grundgesetze (Frege, 1962) are taken from Furth (Frege, 1964) and Geach and Black (1960), respectively. I shall, without comment, change Furth's "course-of-values" (Wertverlauf) to "value-ranges" in order to unify terminology. Further references to Grundgesetze will be in the text marked GGA followed by a Roman numeral for the volume and a section number (whenever necessary). 
In short, the problem is that Axiom $\mathrm{V}$ fails to introduce a criterion of identity for value-ranges that can account for all possible scenarios so that it cannot fix the reference of value-range names even on the intended interpretation - according to which " $\epsilon f(\epsilon)$ " stands for the value-range of the function $f .{ }^{2}$ This is the case because the law only establishes the criteria for cases in which the value-range of some function is identical to another object which is also explicitly given as the value-range of another function (" $\epsilon f(\epsilon)=\alpha \dot{\alpha}(\alpha)$ "), that is, it says nothing as to whether a mixed-identity statement is to be true or false, and, by "mixed-identity statement", I am referring to identities between value-ranges and any other objects not necessarily given as such ( " $\epsilon f(\epsilon)=q$ "). The fact that, in general, Axiom $\mathrm{V}$ is unable to determine the truth-value of identity statements involving valueranges reveals an alarming semantic problem for Frege's attempt to successfully reduce arithmetic to logic: some sentences have no truth-value at all.

Surprisingly, Frege's solution for this "problem of indeterminacy of reference" is to identify truth-values and value-ranges, which provides him a way of determining mixed-identity statements between the two kinds of logical objects of his formalism. This solution, however, has raised serious doubts in the Fregean literature $^{3}$. First, its restriction to truth-values and value-ranges does not seem to correspond with one of Frege's fundamental principles, namely, the "principle of complete determination". According to this principle, concepts must have sharp boundaries, such that the definition of a predicate must say if it is true for all objects. In other words, what one should expect of a complete determination is that Frege actually determines whether, for example, Julius Caesar is the value-range of a given function or not. Of course, what holds for Caesar naturally holds for all other objects. The second doubt is the usual complaint that its restriction makes Frege's solution unable to solve a "persistent version" 4 of what is known as the Caesar problem, an objection

\footnotetext{
${ }^{2}$ Kemp (2005) remarks that, later, in Über die Grundlagen der Geometrie I, Frege writes that "one can never expect principles or theorems to settle the reference of a word or sign" (Frege, 1903, p. 275). Frege is certainly well aware that it is not possible to determine the reference of value-range names axiomatically either, since the permutation argument (see section 2) already shows that the system will always have unintended models. Indeed, as we shall discuss in sections 2-4, the real problem for Frege appears to be that the indeterminacy of reference of value-range names implies that not all sentences of the system are true or false.

${ }^{3}$ See e.g. Parsons (1965), Dummett (1981), Wright (1983), Moore and Rein (1986), Ricketts (1997), Heck (1997b, 1999), Schirn (2001).

${ }^{4}$ I follow Ruffino's (2002) terminology.
} 
about identity criteria for value-ranges. Since Frege apparently fails to respond to those two objections, many scholars are now convinced that neither the problem of the indeterminacy of reference nor the Caesar problem were solved by Frege in GGA, after all.

In this paper I will address these two questions. First, I argue that in order to have an accurate understanding of the problem of indeterminacy of reference and Frege's restricted solution for it, one should consider his "theory of referentiality", which he explains between the sections 29-31 of GGA. With this in mind, I hold that the restriction of Frege's solution to value-ranges and truth-values is not a violation of the principle of complete determination, at least in the form it takes in the system of GGA. Second, I hold that the problem of indeterminacy of reference is merely a matter of fixing the reference of the value-ranges names of the system, whereas the Caesar problem-as previously stated by Ruffino (2002) - is concerned with the question of establishing the nature of numbers as logical objects. Thus, I claim that there is no analogy between those two problems, as it is typically assumed in the literature. However, before addressing those issues, I will provide a brief review of Frege's procedure for dealing with the problem of the indeterminacy of reference in GGA $\S 10$. This will be the objective of sections 2 and 3 of this paper. In section 4, I will review Frege's theory of referentiality discussed in GGA $§ \S 29-31$ in order to better understand his solution to the indeterminacy. In section 5, I will offer an alternative view of the principle of complete determination into the system of GGA that does not allow any indeterminacy of the reference of value-range names. In section 6, I will argue against what is commonly seen as a persistent version of the Caesar problem in GGA.

\section{The permutation argument}

One might ask why we should take the problem of indeterminacy of reference so seriously in the first place. The simple answer is that many elements of Frege's thought strongly suggest that one should do so. First, the importance of this question is emphasized by the fact Frege defines cardinal numbers as particular value-ranges in GGA (§42) and, according to this, any referential indeterminacy in the notion of value-ranges naturally amounts to an indeterminacy of the same sort in the concept of cardinal number. Second, Frege is usually celebrated as an Arch-Platonist, in the sense that he argues that there are objective facts determining the truths of arithmetic. Thus, numerals should mean something, and, so, refer to one and 
only one object. ${ }^{5}$ Third, since Frege regards sentences as names of truth-values, a proof that every name has a unique reference becomes not only a necessary step for a proof of consistency ${ }^{6}$ (a sentence that refers to exactly one truth-value cannot be both true and false), but, more than anything else, a means of expressing the principle of bivalence that states that every sentence must be either true or false (GGA, II, §56). It is important to mention that in order to fulfill the principle of bivalence one does not need to determine exactly which object is supposed to be the referent of a particular name, since determining the truth-value of every sentence in which this name occur is enough for that. As we shall see in the next section, this is exactly what Frege does when he identifies truth-values and value-ranges: he does not specify a unique reference for e.g. " $\epsilon(-\epsilon)$ " (nor does he determine whether it denotes Julius Caesar or not), rather, his primary concern is to make sure that every sentence of the system where the name " $\epsilon(-\epsilon)$ " occurs has a determinate truthvalue. $^{7}$ In this sense, the indeterminacy of reference of value-ranges is unacceptable mainly because it shows that some sentences of the system have no truth-value.

This being said, I shall now address, in the next section and the remainder of this one, Frege's procedure for facing the problem of indeterminacy of reference. This problem is already established in the initial paragraph of GGA $\S 10$, when Frege realizes that his newly introduced axiom to govern the notion of value-ranges, Axiom V (GGA, §3), does not determine the reference of all sentences involving value-range names. The problem is put quite clearly in Frege's own words:

Although we have laid it down that the combination of signs " $\epsilon f(\epsilon)=\alpha g(\alpha)$ " has the same denotation as " $(\forall x)(f(x)=g(x)) "$, this by no means fixes completely the denotation of a term like " $\epsilon f(\epsilon)$ ". We have only a means of always recognizing a value-range if it is designated by a term like " $\epsilon f(\epsilon)$ ", by which it is already recognizable as a value-range. But we can neither decide, so far, whether an object is a value-range that is not given us as such, and to what a function it may correspond, nor decide in general whether a given value-range has a given property unless we know that this property is connected with a property of the corresponding function. (GGA, $§ 10)$

\footnotetext{
${ }^{5}$ Cf. Grundlagen der Arithmetik, $§ 557-61$.

${ }^{6}$ See Sluga (1980, p. 167).

${ }^{7} \mathrm{I}$ am grateful to an anonymous referee for pointing this out to me.
} 
That is, Axiom $\mathrm{V}$ is insufficient to determine the reference (truthvalue) of sentences of the form " $\epsilon f(\epsilon)=q$ ", which amounts to the indeterminacy of the reference of value-range names, since it does not provide us with a general means of recognizing a value-range as the same again when it is not given by the description "the value-range of ...". 8

In order to confirm his informal remarks, Frege articulates a technical argument to confirm the indeterminacy. This so-called "permutation argument" 9 goes as follows:

(i) Let $\Delta$ be an assignment of objects to value-range names in such a way that it satisfies Axiom $\mathrm{V}^{10}$

(ii) Let $h$ be a nontrivial permutation of all objects in the domain of first-order variables, so that for some value-range $a, h(a) \neq a ;^{11}$

(iii) Now consider a new assignment $\Delta^{\prime}$ which is related to $\Delta$ as follows: if $\Delta$ assigns an object $x$ to a value-range name, $\Delta^{\prime}$ will assign $h(x)$ to it;

(iv) Since $h$ is a nontrivial permutation, it follows that at least one value-range name can have either $x$ or $h(x)$ as its reference, without the identity $x=h(x)$ assured.

In other words, the permutation argument shows that if there is an interpretation that satisfies Axiom V, then, by permutation, there will be others, so that the reference of the value-range names is not uniquely determined by Axiom $\mathrm{V}$.

\footnotetext{
${ }^{8}$ Surprisingly, not all cases of indeterminacy are strictly of the form " $\epsilon f(\epsilon)=$ $q$ ". Recall that Frege allowed value-range names for functional expressions of

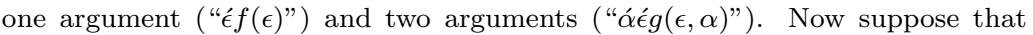

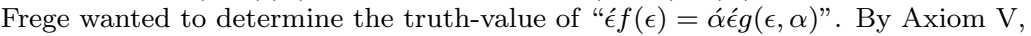
this sentence is co-referential (gleichbedeutend) with " $\forall x)(f(x)=\dot{\epsilon} g(\epsilon, x))$ ". However, we know that Axiom $\mathrm{V}$ cannot fix its reference if $f(a)$ is a truth-value for some $a$. In sum, Axiom $\mathrm{V}$ might even fail to fix the reference of nonmixedidentity statements such as " $\epsilon f(\epsilon)=\alpha \dot{\epsilon} g(\epsilon, \alpha)$ "-I am indebted to Alessandro Duarte for reminding me of this point. Thus, it is perhaps more accurate to state that Axiom $\mathrm{V}$ is unable to decide the truth-value of sentences which are of the form " $\epsilon f(\epsilon)=q$ " up to sameness of reference (Bedeutungsgleichheit).

${ }^{9}$ Dummett (1981, p. 408).

${ }^{10} \mathrm{I}$ assume here, for the sake of argument, that Axiom $\mathrm{V}$ is consistent.

${ }^{11}$ It is uncontroversial in the literature whether this permutation should be expressible in the formal language of GGA or not. Dummett (1981) claims that it does not. Moore and Rein (1986, p. 378) argue that it does, pointing out that this perhaps explains Frege's additional remark "at least if there does exist such a function..." (GGA, §10).
} 


\section{The identification of value-ranges and truth-values}

According to Frege, it is possible to solve the indeterminacy simply by "its being determined for every function when it is introduced, what values it takes on for value-ranges as arguments, just as for all other arguments" (GGA, §10). As a result, he limits himself to the only three functions introduced in his formalism up to this point:

(i) " $-x$ " (the horizontal): the function whose value is the True for the True as argument and the False otherwise;

(ii) " $\neg x$ " (negation): the function whose value is the True for the False as argument and the False otherwise;

(iii) " $x=y$ " (identity): the function whose value is the True if $x$ refers to the same object as $y$ and the False otherwise.

Next, Frege observes that if it is determined what values identity has for value-ranges as arguments, the horizontal and negation will be determined as well. This holds for two reasons: (i) since the value of the function " $x=x$ " is the True for all arguments, the horizontal can be expressed through identity as the function " $x=$ $(x=x)$ "; (ii) since we can understand " $\neg \Delta$ " as the negation of " $-\Delta$ " (i.e. " $\neg(-\Delta)$ "), negation can be seen as a function applied to the horizontal. Hence Frege's claim that to determine what values identity has for value-ranges as arguments is the same as determining them for the horizontal and negation.

However, it is worth mentioning that Frege's starting point was the very problem that Axiom $\mathrm{V}$ was insufficient to determine the truth-value of sentences of the form " $\epsilon f(\epsilon)=q$ ", so we are incurring circularity by trying to solve it by determining what values identity has for value-ranges as arguments. Frege's way out of this impasse is pointed out by the very permutation argument: recall that it shows that Axiom $\mathrm{V}$ is incapable of determining a unique reference to an arbitrary value-range name " $\epsilon f(\epsilon)$ ", so it does not rule out the possibility of the referent of " $\epsilon f(\epsilon)$ " being any object in the domain, including truth-values. To put it another way, this shows that it is consistent with Axiom $\mathrm{V}$ for value-ranges to be truth-values:

(i) Let $\Delta$ be an assignment of objects to value-range names in such a way that it satisfies Axiom V;

(ii) Let $f$ and $g$ be two noncoextensional functions and $a$ and $b$ be the objects denoted by " $\epsilon f(\epsilon)$ " and " $\epsilon g(\epsilon)$ ", respectively; 
(iii) Let $h$ be the following nontrivial permutation of all objects in the domain of first-order variables:

$$
h(x)= \begin{cases}\text { True } & \text { if } x=a \\ a & \text { if } x=\text { True } \\ \text { False } & \text { if } x=b \\ b & \text { if } x=\text { False } \\ x & \text { otherwise }\end{cases}
$$

(iv) Now consider a new assignment $\Delta^{\prime}$ which is related to $\Delta$ as follows: if $\Delta$ assigns an object $x$ to a value-range name, $\Delta^{\prime}$ will assign $h(x)$ to it instead;

(v) It follows without contradicting Axiom $\mathrm{V}$ that it is possible to identify the value-ranges of two arbitrary noncoextensional functions $f$ and $g$ with the True and the False. ${ }^{12}$

(Note that $f$ and $g$ must be noncoextensional, otherwise (v) would contradict the premise that the True and the False are different objects.)

Frege then feels free to stipulate that some value-ranges are truth-values. Unsurprisingly, he chooses the function "same as the True", the horizontal, to play the role of $f$ and defines a function "same as the False", " $y=(\forall x)(\neg(x=x))$ ", to play the role of $g$, stipulating: (T) " $\epsilon(-\epsilon)$ " denotes the True and (F) " $\epsilon(\epsilon=(\forall x)(\neg(x=x)))$ " denotes the False. And now, with $(\mathrm{T})$ and (F) as supplements to Axiom $\mathrm{V}$, it is possible to determine the truth-value of mixed-identity statements between value-ranges and truth-values, so that Frege believes to have determined the reference of the value-range names as far as it is possible at this point of the construction of the system (whenever we introduce new functions that are not reducible to the old ones the same problem appears again).

\section{Frege's criteria of referentiality}

Before proceeding any further, I would like to examine Frege's claim that in order to solve the indeterminacy, it suffices to

\footnotetext{
${ }^{12}$ This stipulation is sometimes called the "trans-sortal identification" (Dummett, 1991) or "identifiability thesis" (Schroeder-Heister, 1987) in the literature. Its acceptance among Fregean scholars, however, is not univocal. Schroeder-Heister (1987) argues against its arbitrariness saying that that the permutation argument provides no justification for the identification. For a reply to Schroeder-Heister's criticism, see Moore and Rein (1987), especially p. 52.
} 
determine for each function what values it has for value-ranges as arguments. It is by no means obvious why we should overcome the indeterminacy this way, which has led to some debates in the Fregean literature. As far as I am aware, the most accepted views are Dummett's suggestion of the procedure as a generalized version of Frege's context principle, that is, the principle that states that only in the context of a sentence does a name have a reference, and Ruffino's reading of the procedure as an application of Leibniz's principle, namely, the famous logical principle that two objects are identical if and only if every function has the same value for both objects as arguments. ${ }^{13}$ However, I do not share either of these views. What both Dummett and Ruffino have to take into account is that Frege's procedure is deeply connected with the "criteria of referentiality" that he discusses in sections $29-31$ of GGA. ${ }^{14}$ This is where he attempts to provide a proof that every value-range name is referential (that is, that it has a reference). And, taking Frege's actual account of referentiality, it is possible to understand that his procedure in GGA $\S 10$ is just an application of a formalized version of the principle of complete determination, that is, the principle that says that for a predicate to be adequately defined in science, it should be determined under which circumstances it is true or not of all objects ${ }^{15}$. I shall further develop this view in the remainder of this section and the following section.

Frege's proof that every value-range name has a reference is ultimately found in his famous "proof of referentiality" - a demonstration that all the names of the formalism are referential. The proof itself is carried out in GGA $\S 31$, but its fundaments are presented earlier in the text. For instance, since only well-formed names can be ascribed a reference, section 30 explains the formation rules of Frege's formalism, where a key element of the proof will be the fact that there are only names for value-ranges and truth-values in the formalism, that is, there are not names for urelements - objects (concrete or abstract) that are not sets (value-ranges). There are some other interesting details about Frege's method of forming names, ${ }^{16}$ but I think I have given

\footnotetext{
${ }^{13}$ Cf. Dummett (1981, p. 408) and Ruffino (2002, pp. 128-130).

${ }^{14}$ Since Frege's theory of referentiality is presented in $\S \S 29-31$, offering a justification for Frege's strategy of determining the reference of value-range names in $\S 10$ without taking $§ \S 29-31$ into account (as Dummett and Ruffino seem to do) is to erroneously regard $\S 10$ as if it were a section that is isolated and disconnected from the rest of GGA (see Heck (1999, p. 258)). In fact, as discussed at the end of this section, nothing but Frege's theory of referentiality is needed to justify his strategy in $\S 10$.

${ }^{15}$ See GGA II, §57.

${ }^{16}$ Cf. Bentzen (2014, p. 61).
} 
enough information here to enable me to present the proof I would like to discuss.

The idea behind Frege's proof of referentiality is that (i) the primitive names of the system are referential and (ii) the referentiality of names composed by primitive names follows by induction. What I want to examine in detail is the way Frege elaborates the steps of his proof. For the sake of clarity, I will discuss the latter first.

(ii) Frege begins the argument for his proof in section 29, where he articulates its inductive step by establishing for each syntactic type of name in his formalism what I call a "criterion of referentiality", which is a particular requirement for ascribing proper names and functional expressions a reference:

(a) A first-level functional expression of one argument " $f(x)$ " is referential if the proper name " $f(\Delta)$ " that results from the application of " $f(x)$ " to " $\Delta$ " always has a reference for any referential proper name " $\Delta$ ";

(b) A proper name " $\Delta$ " is referential if (i) the proper name " $f(\Delta)$ " that results from " $f(x)$ ", when its argument-places are filled by " $\Delta$ ", always has a reference for any referential first-level functional expression of one argument " $f(x)$ "; (ii) the first-level functional expression of one argument " $f(\Delta, y)$ " that results from " $f(x, y)$ ", when its $x$-argument-places are filled by " $\Delta$ ", always has a reference for any referential first-level functional expression of two arguments " $f(x, y)$ ", and if the same holds for its $y$-argument-places as well;

(c) A first-level functional expression of two arguments " $f(x, y)$ " is referential if the proper name " $f(\Delta, \Gamma)$ " that results from " $f(x, y)$ ", when its $x$ - and $y$-argument-places are respectively filled by " $\Delta$ " and " $\Gamma$ ", always has a reference for any referential proper names " $\Delta$ " and " $\Gamma$ ".

Note that Frege's criteria simply state that a name is referential if and only if it yields new referential names when it takes other referential names in the name formation.

(i) Whereas Frege's goal in section 31 is to show that all primitive names of his formalism are referential, it is enough to limit myself to his account of quantifiers and, of course, value-range names here. One might ask what, in principle, quantifiers have to do with the indeterminacy of value-range names. To answer this question, take, for instance, the following sentence definable in GGA's formalism:

$$
(\exists x)(\forall F)(\neg(x=\hat{\epsilon} F(\epsilon)))
$$


Note that it essentially says that "there is at least one object that is not a value-range of a function". Is this sentence true or false? Since the domain of GGA is usually believed to be all-inclusive, such that first-order variables should range over all possible objects, it seems plausible to claim that this sentence should be true: after all, there are several counterexamples of objects in the all-inclusive universe that are not value-ranges-people, trees, planets (i.e. urelements). ${ }^{17}$ Indeed, it is worth mentioning that there is some evidence suggesting that Frege did attempt to do something like identifying urelements with value-ranges. This issue is discussed in a famous footnote to section 10, a passage that drew the attention of several commentators due to its connection to Frege's commitment to the principle of complete determination. The reason for this is that this footnote is the place where Frege attempts to generalize his restricted solution for the indeterminacy (from truth-values and value-ranges) to all possible objects in general. Recall that Frege's solution to the indeterminacy was to stipulate $(\mathrm{T})$ and $(\mathrm{F})$, which consists of identifying the True with the value-range of the function "same as the True" and the False with the value-range of the function "same as the False". Now, a natural generalization of it would be to identify every object $a$ with the value-range of the function "identical to $a$ ", that is, to stipulate:

$$
\Delta=\dot{\epsilon}(\epsilon=\Delta)
$$

which basically means to identify every object with the value-range of a property only satisfied by itself and no other object, a procedure similar to identifying an object with its singleton. However, this proposal was rejected, for in the case where $\Delta$ is already known to be a value-range of some function $f$, we would have the following instance

$$
\alpha f(\alpha)=\dot{\epsilon}(\epsilon=\dot{\alpha} f(\alpha))
$$

which, by Axiom $\mathrm{V}$, has the same truth-value as

$$
(\forall x)(f(x)=(x=\alpha f(\alpha)))
$$

which is false when $f$ maps an argument other than $\alpha f(\alpha)$ itself to the True.

The point to be made here, however, is: why did Frege attempt to provide a general solution to the problem of indeterminacy in the first place? Didn't he claim his restricted solution was enough to fix

\footnotetext{
${ }^{17}$ See Dummett (1981), Heck (1999), Schirn (2001), among others. In fact, in no part of GGA Frege is clear about what objects his first-order variables are supposed to range over.
} 
the reference of value-range names (as far as this is possible)? The fact that Frege's general solution failed to solve the indeterminacy served as the root of the suspicions about the legitimacy of Frege's restricted solution in the Fregean literature. For the time being, I shall return to the analysis of (1), leaving discussion of criticisms of Frege's restricted solution for sections 5 and 6 .

I have exposed enough reasons to claim that (1) is true. And, in fact, this is the view of several influential commentators in the Fregean literature. ${ }^{18}$ However, an ultimate answer for this issue should also rely on Frege's interpretation of quantifiers. At first glance it may seem that Frege subscribes to a substitutional interpretation of quantifiers in GGA (Resnik, 1986; Dummett, 1991; Hintikka and Sandu, 2005), that is, the view that a quantified sentence is true if and only if all of its substitution instances are true:

To investigate whether the name " $(\forall x)(\phi(x))$ " of a second-level function denotes something, we ask whether it follows universally from the fact that the function-name " $f(x)$ " denotes something, that " $(\forall x)(f(x))$ " succeeds in denoting. Now " $f(x)$ " has a denotation if, for every denoting proper name " $\Delta$ ", " $f(\Delta)$ " denotes something. If this is the case, then this denotation either always is the True (whatever " $\Delta$ " denotes), or not always. In the first case " $\forall x)(f(x))$ " denotes the True, in the second the False. Thus it follows universally from the fact that the substituted function-name " $f(x)$ " denotes something, that " $(\forall x)(f(x))$ " denotes something. Consequently the function name " $\forall x)(\phi(x))$ " is to be admitted into the sphere of denoting names. (GGA, §31)

However, this is very unclear, since Frege appears to vacillate between the substitutional and objectual interpretation in GGA (Martin Jr , 1982). In fact, over the last few years, Linnebo (2004) has offered strong arguments that Frege's strategy in $§ \S 29-31$ is neither compatible with a substitutional nor objectual interpretation of quantifiers. Linnebo proposes instead a reading of Frege's view on quantifiers based on Heck's "auxiliary names" (Heck, 1997b), a special category of names corresponding to Frege's uppercase Greek letters that essentially serves the same purpose as assignments of values to free variables do in modern

\footnotetext{
${ }^{18}$ See e.g. Dummett (1981, ch. 15), Heck (1999, pp. 272-273), Schirn (2001, pp. $45-46)$.
} 
logic. Under this view, it follows that (1) is true iff the domain of GGA is not limited to truth-values and value-ranges as objects. More importantly, this interpretation raises the question of whether Frege's referentiality criteria should also be interpreted in terms of auxiliary names or not. As Linnebo (2004) points out, the former view will say that a candidate name is referential just in case all names which are formed by combining the candidate name with a referential name of the correct syntactic category (whether this name is part of the language or not) are referential; the latter view, the nonauxiliary one, will say that a candidate name is referential just in case it forms referential names when correctly combined with names already recognized as referential. Following Linnebo, I shall favor the latter view, which has been supported by powerful arguments. $^{19}$

With that said, I can now return to Frege's proof. In the case of value-range names, Frege's proof must be more involved because until the introduction of value-range names all the previous existing names of GGA are sentence names (names of truth-values), which means that he is introducing names which stand for a new category of objects, namely, value-ranges. Now, since value-range names fall into the category of proper names, one must apply the criterion (b) stated in 4.(ii) for proper names in general. Under the nonauxiliary reading, (b) can be reformulated as follows:

$\left(\mathrm{b}^{*}\right)$ Let " $f(x)$ " be a functional expression already recognized as referential. A value-range name " $\epsilon f(\epsilon)$ " is referential if (i) the proper name " $g(\hat{\epsilon} f(\epsilon))$ " that results from " $g(x)$ ", when its argument-places are filled by " $\epsilon f(\epsilon)$ ", always has a reference for any first-level functional expression of one argument " $g(x)$ " already recognized as referential; (ii) the first-level functional expression of one argument " $g(\epsilon f(\epsilon), x)$ " that results from the filling of the $x$-argument-places of " $g(x, y)$ " with " $\epsilon f(\epsilon)$ " always has a reference for any first-level functional expression of two arguments " $g(x, y)$ " already recognized as referential, and if the same holds for its $y$-argument-places as well.

In short, Frege's criterion of referentiality states that in order to determine if a proper value-range name (value-range names formed from functional expressions already recognized as referential) is referential, it suffices to determine for each first-level functional expression $g$ already recognized as referential what values it has for

\footnotetext{
${ }^{19}$ See Linnebo $(2004, \S \S 4-6)$.
} 
this value-range as argument. However, we should recall the following quotation from the section 10:

How may this indefiniteness be overcome? By its being determined for every function when it is introduced, what values it takes on for value-ranges as arguments, just as for all other arguments. (GGA, §10)

A careful examination shows that this is the very strategy Frege designed earlier in GGA $\S 10$ as a way out of the indeterminacy of the value-ranges. That is, there is no reference to any generalized context principle, and no Leibniz principle is invoked in this passage. In fact, this interpretation is confirmed once again in the closing passage of GGA $\S 10$ :

With this we have determined the value-ranges so far as is here possible. As soon as there is a further question of introducing a function that is not completely reducible to functions known already, we can stipulate what value it is to have for value-ranges as arguments; and this can then be regarded as much as a further determination of the value-ranges as of that function.

In sum, nothing but Frege's own account of the referentiality is used to explain his suggestion to solve the indeterminacy by determining for every function what values it has for value-ranges as arguments in section 10 .

So how Frege's referentiality proof for value-range names is carried out? His argument begins by showing that when proper value-ranges are placed in the (a) $x$-argument places of the functional expressions of one argument " $-x$ " and " $\neg x$ " they yield a referential proper name; (b) $x$-argument or $y$-argument places in the functional expressions of two arguments " $x=y$ " and " $(-x) \rightarrow(-y)$ " (the conditional) they yield a referential functional expression of one argument. He then remarks that " $(-x) \rightarrow(-y)$ " is referential only if the names " $-x "$ and " $-y$ " are referential. Now, since we already know that the horizontal and negation are reducible to identity, it turns out that a (proper) value-range name " $\epsilon f(\epsilon)$ " is referential if and only if both " $\epsilon f(\epsilon)=y$ " and " $x=\epsilon f(\epsilon)$ " are referential functional expressions of one argument. And, with this, he attempts to justify his solution in GGA $\S 10$ :

By our stipulations, that " $\epsilon f(\epsilon)=\alpha \dot{\alpha}(\alpha)$ " is always to have the same denotation as " $\forall x)(f(x)=g(x))$ ", that " $\epsilon(-\epsilon) "$ is to denote the True, and that 


\begin{abstract}
" $\epsilon(\epsilon=(\forall x)(\neg(x=x))$ " is to denote the False, a denotation is assured in every case for a proper name of the form " $\Gamma=\Delta$ ", if " $\Gamma$ " and " $\Delta$ " are proper value-range names or names of truth-values. (GGA, $\S 31)$
\end{abstract}

Accordingly, it seems reasonable for Frege to claim to have fixed the reference of value-range names in GGA, once identity - the only remaining case of indeterminacy - is completely determined with the Axiom V supplemented by the restricted stipulation. If there are any questions that should be raised about his procedure, they should be certainly concerning quantified sentences, or more accurately, the denotation of auxiliary names. For if $\Delta$ is an auxiliary name in " $f f(\epsilon)=\Delta$ ", the latter is a referential functional expression only if $\Delta$ denotes a truth-value or value-range. But, being an auxiliary name, $\Delta$ is only subject to the condition that it should denote some object in the domain. ${ }^{20}$

\title{
5 The principle of complete determination
}

In the previous section, I raised some questions regarding Frege's commitment to the principle of complete determination in GGA. Shouldn't the fact that Frege's view on the referentiality of valuerange names does not take into account urelements as objects be

20 This fact has led some scholars such as Heck (1999) and Ruffino (2002) to question whether Frege wasn't operating in a domain restricted to valueranges and truth-values after all. Heck (1999, p. 272) considers this possibility, but quickly argues against it, under the allegation that Frege sharply criticizes other logicians who talk of restricted domains - Heck is referring here to Frege's objection in GGA II $\S 65$, which, as he admits, is the only passage he found in which Frege speaks directly against this possibility (cf. Heck (1999, fn. 25)). In section 5, I shall offer a novel interpretation of Frege's objection that is not inconsistent with this restriction of the domain. Ruffino remarks that, when discussing the range of objects of his theory, Frege writes that "I count as objects everything that is not a function, for example, numbers, truth-values, and the value-ranges" (GGA, §2), all of which are fundamentally value-ranges. Nevertheless, Ruffino (2002, pp. 137-138) is aware that this is far from being a decisive piece of textual evidence to support his claim. In any case, it appears that there is no option but to suppose that an earnest thinker such as Frege wouldn't consider other objects such as urelements without explicitly saying so-especially in such a critical section such as GGA §31. Indeed, if one refrains from committing to this view that the domain of the system of GGA is restricted to value-ranges and truth-values, the only alternative left that seems to explain Frege's lack of concern about urelements is to admit a nonauxiliary interpretation of his quantifiers. However, as I mentioned before, this reading is very problematic. Fortunately, this speculative discussion about the domain of GGA will not be relevant to my main arguments in this paper and, for this reason, I shall not address this issue here. 
seen as an explicit violation of principle of complete determination in GGA ${ }^{21}$ For instance, in the following passage of GGA II, Frege explicitly criticizes a similar account of referentiality restricted to numbers as objects:

But can we not stipulate that the expression 'the sum of one object and another object' is to have a reference only when both objects are numbers? In that case, you may well think, the concept something that gives the result one when added to itself is one with sharp boundaries; for now we know that no object that is not a number falls under it. E.g. the Moon does not fall under it, since the sum of the Moon and the Moon is not one. This is wrong. On the present view, the sentence 'the sum of the Moon and the Moon is one' is neither true nor false; for in either case the words 'the sum of the Moon and the Moon' would have to stand for something, and this was expressly denied by the suggested stipulation. (GGA, II, §64)

Indeed, Frege's objection seems to imply that it is a mistake to think that the concept "something that is a value-range of some function" has sharp boundaries in GGA, because its corresponding functional expression

$$
(\exists F)(x=\hat{\epsilon} F(\epsilon))
$$

only has a reference for value-ranges and truth-values as objects; that is, (4) has no reference at all for urelements as objects, meaning that no complete determination in this sense takes place in GGA. ${ }^{22}$ What's more, there is no doubt that Frege believed that his proof in GGA $\S 31$ indeed suffices to guarantee a reference for each value-range name, which confirms that, according to him, the reference of valuerange names is completely determined by his restricted account. ${ }^{23}$

Is it possible that Frege simply abandoned the principle of complete determination during some part of the book? This seems highly unlikely. Frege is well-known for his wish to establish arithmetic in a solid basis as an exact science, and, according to

${ }^{21}$ See, for instance, Heck (1999).

${ }^{22}$ This is substantially the position of many Fregean commentators such as Parsons (1965), Dummett (1981), Wright (1983), Ricketts (1997), Heck (1997b, 1999, 2005), Schirn (2001).

${ }^{23}$ At least until his acknowledgment of Russell's paradox, "It seems, then, [...] that my explanations in $\S 31$ are not sufficient to ensure that my combinations of signs have a meaning in all cases." (Frege, 1967a) 
him, only the principle of complete determination can settle the guidelines of the proper scientific use of a name or predicate:

So long as it is not completely defined, or known in some other way, what word or symbol stands for, it may not be used in an exact science [...] (GGA, II, §57)

More importantly, Greimann (2003) recently pointed out an interesting passage in Frege's writings where he states explicitly the role of the criteria of referentiality in GGA and its relation with the principle of complete determination:

Here again we likewise see that the laws of logic presuppose concepts with sharp boundaries, and therefore also complete definitions for names of functions, like the plus sign. In vol. I we expressed this as follows: every function-name must have a reference. Accordingly all conditional definitions, and any procedure of piecemeal definition, must be rejected. Every symbol must be completely denied at a stroke, so that, as we say, it acquires a reference. (GGA, II, §65)

With this in mind, it is natural to take the criteria of referentiality as the formal incorporation of the principle of complete determination in the system of GGA, and the proof of referentiality as the demonstration that all predicates and proper names of the system can be properly used in science, which basically means that the references of all names in the formalism are completely determinate.

Nevertheless, it should be noted that, under the nonauxiliary reading, Frege's criteria of referentiality do not imply that, say, " $f(x)$ " is referential if, for every object $a$, it follows that " $f(a)$ " denotes something. ${ }^{24}$ Instead, they suggest that " $f(x)$ " is referential if, for every meaningful name $\Delta$ expressible in the formal language of the system, it follows that " $f(\Delta)$ " denotes something. Therefore, it appears that the principle is represented linguistically in GGA. ${ }^{25}$

Besides, because there are no names for urelements, it seems somehow acceptable for Frege that, in the system of GGA, the principle of complete determination neglects urelements and applies only to value-ranges and truth-values, the objects on which he intends to construct his arithmetic. But if this is the case, what

${ }^{24}$ For an auxiliary name ' $a$ ' denoting $a$.

${ }^{25} \mathrm{~A}$ similar conclusion is drawn by Greimann (2003, pp. 273-275). 
would be Frege's motivation to generalize his stipulation in the footnote to section 10? There is nothing wrong with this, if it is for mere convenience, that is, to generally solve any indeterminacy that would arise from adding new names to the language. As well-known, Frege thinks of his formalism as a lingua characteristica in the Leibnizian sense. Therefore, his symbolic notation for concepts should be suitable not only for arithmetic, but, in principle, for discussing any other domain, such as geometry or physics, as he suggests in the preface of Begriffsschrift (Frege, 1879). ${ }^{26}$

So Frege subscribed to a principle of complete determination limited to value-ranges and truth-values in GGA. That said, what could be the point of his objection in GGA II $\S 64$ ? In the following section of GGA II, Frege raises another similar objection, which, as he says, attempts to "throw light on the matter from other sides." (GGA, II, §65)

If anybody wants to exclude from consideration all objects that are not numbers, he must first say what he takes 'number' to mean, and then further extension of this term is inadmissible. Such a restriction would have to be incorporated in the definition, which would thus take some such form as: 'If $a$ and $b$ are numbers, then $a+b$ stands for ...' We should have a conditional definition. [...] By a well-known law of logic, the proposition 'if $a$ is a number and $b$ is a number then $a+b=b+a$ ' can be transformed into the proposition 'if $a+b$ is not equal to $b+a$ and $a$ is a number, then $b$ is not a number' and here it is impossible to maintain the restriction to the domain of numbers. (GGA, II, §65)

Frege's principle of complete determination, in the system of GGA, applies to only value-ranges and truth-values (henceforth

\footnotetext{
${ }^{26}$ This brings the question of how the nonlogical axioms of a scientific theory with urelements may be accommodated in the system of GGA. Because scientific theories often favor the use of urelements, the system of GGA could not be straightforwardly used as the logical part of an axiomatized scientific theory. I suspect that, after the failure of his proposal in the footnote to section 10 , Frege was well-aware of this fact. But I do not believe this prevents the usage of the system of GGA as a lingua characteristica. Suppose that Frege wanted to augment the language of GGA with names for point particles and the conventional postulates of classical mechanics, for example. In order to guarantee that the resulting theory could be properly used as a scientific theory, Frege could try to expand his proof of GGA $\S \S 29-31$ with additional stipulations such that (i) none of the point particles names refer to a value-range and (ii) every sentence name corresponding to a nonlogical axiom refers to the True.
} 
referred to as logical objects). While every number is a value-range of some particular function (GGA, §42), certainly not all value-ranges are numbers. From this viewpoint, Frege's objections are indeed coherent with his referentiality criteria: one is not allowed to think that concepts such as "something such that the sum of it and itself equals 1" have sharp boundaries if they are only "completely" determined for numbers as objects, because many other logical objects are left out of account. For instance, the sentence "the sum of the True and the True equals 1" would lack a reference, and the same holds for most value-ranges as well.

In sum, we can conclude that, according to Frege, one simply cannot neglect logical objects when completely determining a concept in the system of GGA, unlike urelements, which may always be left out of account without any problems. ${ }^{27}$ To put it another way, logical objects seem to have a privileged role in GGA, which comes as no surprise, considering Frege's conviction that no other objects are needed in a logicist foundation of arithmetic.

\section{The Caesar problem}

Finally, I would like to make two points about what seems to be the last objection going against my suggestion that it seems acceptable for Frege to apply the principle of complete determination only to logical objects in GGA. What is widely known as the "Caesar problem" in the Fregean literature is the most decisive piece of textual evidence in Frege's writings supporting the relevance of urelements in the determination of the reference of a name or predicate for the proper use in science. The Caesar problem takes place in Frege's earlier book, Grundlagen der Arithmetik (GLA), an investigation about the logical nature of the concept of number. In fact, before his actual definition of individual numbers as value-ranges in GGA, Frege's most prominent attempt was to introduce the concept of cardinal number via the following identity criterion (Hume's Principle): the

\footnotetext{
${ }^{27}$ A similar conclusion is drawn by Moore and Rein (1986), who claim that Frege restricted his attention to logical objects because "he was concerned only with questions that could be stated within his formalism"; besides, since the language of GGA "contained proper names only for value-ranges and the two truth-values, questions involving other objects (if there are such objects) cannot be formulated within the system." (Moore and Rein, 1986, p. 384, fn. 9). Unfortunately, a problem with Moore and Rein's view is that it cannot explain why Frege chose to do so and deliberately left urelements out of account. I show how this restriction is compatible with my interpretation in section 6 . What's more, Moore and Rein's account lack a justification for Frege's objections in GGA II $\S \S 64-65$, which I see as another benefit of my interpretation.
} 
number of $F$ s is the same as the number of $G$ s just in case $F$ and $G$ are in a one-to-one correspondence. In symbols:

$$
N_{x} F x=N_{x} G x \leftrightarrow F 1-1 G
$$

The reason why Frege did not carry on this definition to GGA is due to the Caesar problem, a problem of indeterminacy concerning the concept of number, namely, the objection that (HP) fails in deciding whether, say, Julius Caesar is identical to the number of moons of Jupiter:

In the proposition [the number of $F \mathrm{~s}$ is identical with the number of $G \mathrm{~s}$ ] the [number of $F \mathrm{~s}$ ] plays the part of an object, and our definition affords us a means of recognizing this object as the same again, in case it should happen to crop up in some other guise, say as [the number of $G \mathrm{~s}$ ]. But this means does not provide for all cases. It will not, for instance, decide for us whether [Julius Caesar] is the same as [the number of moons of Jupiter] - if I may be forgiven an example which looks nonsensical. Naturally no one is going to confuse [Julius Caesar] with [the number of moons of Jupiter]; but this is no thanks to our definition of [number]. ${ }^{28}$ (Frege, $1884, \S 66)$

Although Frege's inept words look quite nonsensical at first glance, the usual consensus in the Fregean scholarship is that his objection reveals a deep concern about four inter-related dimensions of fundamental problems: ${ }^{29}$

(i) The epistemic problem: (HP) does not afford us a means of recognizing numbers as the same again if they are not given as number names. It is now well known that (HP) together with second-order logic is able to prove Peano's postulates - a result called Frege's Theorem. ${ }^{30}$ However, the resulting

\footnotetext{
${ }^{28}$ This passage is transposed from the context of Frege's original discussion about an analogous principle governing the notion of directions (although certainly not a similar principle in the sense that directions are not supposed to be logical objects).

${ }^{29}$ As far as I am aware, Heck (1997a) was the first to notice that the Caesar problem poses both an epistemic and a semantic objection. Later, Greimann (2003) reinforced Heck's view and added to it ontological and logical dimensions. More recently, MacBride (2006) distinguished ontological, epistemic and semantic aspects of the problem, as well, but he seems to have overlooked its logical dimension.

${ }^{30}$ Cf. Wright (1983), Boolos (1987).
} 
arithmetic, also known as Frege's arithmetic, cannot truly represent the way we apprehend numbers as objects, once (HP) can't explain our common understanding of numbers. We certainly know that objects such as the Moon or Julius Caesar are not numbers and an adequate definition should provide identity criteria for numbers that settle the falsity of such expected identity statements.

(ii) The ontological problem: (HP) does not inform us what kind of entity numbers really are. The classic example is Zermelo's and von Neumann's definitions of number. The former defined 0 to be the empty set and defined the successor function as a mapping from $x$ to the singleton of $x$, resulting in a progression like $\emptyset,\{\emptyset\},\{\{\emptyset\}\} \ldots$ The latter defined 0 as the empty set as well, but defined the successor function as a mapping from $x$ to the union of $x$ and the singleton of $x$, forming the progression $\emptyset,\{\emptyset\},\{\{\emptyset\},\{\{\emptyset\}\}\} \ldots$ As a result, one has that, for example, "the number of vertices of a triangle $=$ the number of inner angles of a triangle" holds, but from this it does not follow which particular object is the number 3 .

(iii) The logical problem: (HP) does not establish a sharp distinction of the boundaries of the concept of number. (HP) does not introduce properly the predicate " $x$ is a number", since it does not determine completely, for every object, whether this predicate must be applied to it. This objection is closely related to the principle of complete determination, and one can find here some similarity with the core problem discussed in section 5 for value-ranges.

(iv) The semantic problem: (HP) does not fix the reference of sentences containing number names. Frege's doctrine of sense and reference establishes that all sentences must have a truth-value. More precisely, the problem is seen as the technical difficulty that (HP) cannot determine whether the reference of " $N_{x} F x=q$ " is to be the True or the False, therefore not determining the referent of " $N_{x} F x$ ".

Moreover, it can be seen that the epistemic, ontological, and logical problems can be all reduced to the semantic dimension, in the sense that the latter is the most basic of the four objections: (i) When faced with the question of how numbers are given to us in GLA $\S 62$, Frege invokes the context principle, and the investigation takes the form of asking how we can fix the reference of sentences containing number names. First noted by Dummett (1993), this passage is now often regarded as the one that led to the linguistic 
turn in philosophy. (ii) Given Frege's context principle, the matter of establishing which kind of entity numbers are amounts to the task of fixing the reference of number names. (iii) For Frege, once the principle of complete determination settles the guidelines for ascribing a predicate a unique and determinate reference, the logical problem is reduced to the semantic problem, as well.

That said, the first point I would like to make is how Frege's Caesar objection for numbers in GLA seems to anticipate the problem of indeterminacy of reference for value-ranges in the initial paragraph of GGA $\S 10$, as, structurally, both objections are raised in the same way. The usual argument for this goes as follows. ${ }^{31}$ Note that both Axiom $\mathrm{V}$ and (HP) are all instances of the same scheme, what is today known as an abstraction principle ${ }^{32}$

$$
f(a)=f(b) \leftrightarrow a \sim b
$$

where $\sim$ is a reflexive, symmetric, and transitive (i.e. equivalence) relation. Now, it is easy to see that any instance of (AP) is unable to determine whether " $f(a)=q$ " is true or false: one has not enough information to verify if its corresponding equivalence relation holds or not. Therefore, one should see undetermined identity statements which are left by both Axiom V and (HP) as a consequence of a limitation of the very technique of abstraction itself. This is supposed to show not only a close parallel between the problem of indeterminacy of reference and the Caesar problem, but that, in principle, exchanging (HP) for Axiom $\mathrm{V}$ would only "solve" the problem by passing it on from numbers to value-ranges. But - and this is a crucial point - this is exactly what Frege seems to do to solve the Caesar problem in GLA: facing this obstacle with (HP), he simply explicitly defines the operator "the number of Fs" as the "extension of the concept in one-one correspondence with $F$ ", and adds to it a footnote "I assume that it is known what the extension of a concept is" (Frege, 1884, §68). Ultimately, extensions are generalized to the notion of a value-range later in GGA, so that the problem of indeterminacy of reference can be seen as a consequence of his "unfinished business" of GLA and interpreted as a persistent version of the Caesar problem that recurs in GGA for value-ranges. Note that all of this confirms the Caesar problem as a semantic one, characterizing what I would like to call the "strong view" of the Caesar problem, a quite prominent view in the Fregean literature since initially suggested by Parsons (1965), and

\footnotetext{
${ }^{31}$ See e.g. Kemp (2005, p. 183), and Heck (2005, p. 163).

${ }^{32}$ Here I ignore the fact that (HP) is a second-order principle.
} 
subsequently argued by both Dummett (1981) and Wright (1983) several years later.

The second point I would like to stress is the reason I think this strong view is incorrect. The reason I call it a "strong view" is because, when taken literally, that is, as the objection that a definition must decide identity statements between the definiendum and all possible objects, the Caesar problem is too strong. In everyday mathematics, such as in the definition of a topological space, we do not worry about whether Julius Caesar is an open set or not. ${ }^{33}$ The reason we do not care about this is that urelements play no role in conventional mathematics. The universe of set theory - at least in the Zermelo-Fraenkel theory with the axiom of choice (ZFC), usually considered the standard foundational theory of mathematics - is constructed out of sets alone. However, according to the strong view, Frege's objection in this passage suggests that a foundational theory should take urelements in account, at least to assure that the predicates it defines have a precise scientific meaning. This is clearly in contrast with my considerations about Frege's acceptance of the principle of complete determination being restricted to logical objects. But not only this: the strong view poses such a severe objection that even all of Frege's very procedures regarding identities in GGA §]10 and $\S \S 29-31$ are unable to overcome. That is, recall that Frege did not decide in the theory of GGA whether an arbitrary urelement is a value-range or not-and I believe this should be thought as signaling that something must be wrong with the strong interpretation rather than with Frege's very procedures, a frequent claim in the literature. ${ }^{34}$

Therefore, instead of mitigating the shortcomings of the strong view, I would like to consider an alternative interpretation of the Caesar problem due to Ruffino (2002) that has proved faithful to Frege's procedures regarding identities in GGA, while confirming

\footnotetext{
${ }^{33}$ In fact, questions such as this one are pointless or even meaningless for the working mathematician. As Kemp (2005) remarks, "If only in the context of a sentence has a word really a meaning, then why is it not the case that only in the context of a theory or language has a sentence really a meaning?" On the other hand, it appears that such kind of question should be answered negatively, since people and sets are completely different categories of objects. For an account of reference and objecthood that is faithful to both kind of intuitions regarding cross-category identities, see Linnebo (2005).

${ }^{34}$ Cf. e.g. Dummett (1981, p. 408). In fact there is a third possibility, which is to suppose that Frege has changed his approach to the problem from GLA to GGA, so that the strong view may still be a legitimate concern. However, I do not believe this is the case. As we shall see in the remainder of this section, it is very unclear that Frege indeed endorsed the strong view even in GLA.
} 
my suggestion about Frege's relaxed view on the principle of the complete determination - at least in respect to urelements. This view, I think, also helps us to clarify Frege's motivation for the introduction of extensions of concepts in GLA, as argued as follows. The initial point that deserves to be highlighted is that in some passages of Frege's writings, he actually suggests that value-ranges play a special role for him in his attempt to reduce arithmetic to logic. This has been first observed by Ruffino, who recalls an explicit quote of Frege's favoured treatment of value-ranges from a letter to Russell dated July 28, 1902:

I myself was long reluctant to recognize value-ranges and hence classes; but I saw no other possibility of placing arithmetic on a logical foundation. But the question is, how do we apprehend logical objects? And I have found no other answer to it than this, we apprehend them as extensions of concepts, or more generally, as value-ranges of functions. (Frege, 1967b, pp. 140-141)

This passage sheds light on many aspects of GLA. First, it reveals that (HP) is rejected in the book because, in Frege's view, only extensions of concepts can assure the logical nature of the numbers. ${ }^{35}$ But why is this so? This is a very interesting piece of information, since the main argument for the rejection of (HP) in the book is the problem that it cannot decide whether the Roman emperor is the number of some concept or not. Therefore, it is

\footnotetext{
${ }^{35}$ This was also pointed out by Kemp (2005, p. 196). This interpretation seems to be incompatible with Frege's confusing concluding remark in GLA §107: "I attach no decisive importance even to bringing in the extensions of concepts at all." According to Landini (2012), what Frege attaches decisive importance to in GLA is the fact that two concepts can be put in a one-to-one correspondence:

"The mathematical notion of an "extension," popular at the time Frege wrote Grundlagen, was the notion of a class or aggregate of entities. In his Grundlagen, Frege explicitly petitioned his readers to help themselves to this familiar mathematical notion of the extension of a concept (a notion we now call the "logical notion of a class"). He thought this may help in their understanding. But, he later explains, he attaches no decisive importance to the notion of extensions in this sense." (Landini, 2012, p. 115)

Frege's lack of clarity about extensions of concepts in GLA no doubt suggests that he was uncertain about it during that period. In fact, Burge (1984) reminds us of a now lost manuscript from the period immediately after the publication of GLA where Frege apparently attempted to explain what an extension of a concept is and considered an alternative contextual definition for the operator "the number of Fs". Nevertheless, it seems to me that Frege's early struggle against extensions of concepts just provides more evidence that supports his confession to Russell almost two decades later: "And I have found no other answer to it than this, we apprehend [logical objects] as extensions of concepts [...]" (Frege, 1967b, p. 140).
} 
more plausible that the Caesar problem is raised in GLA only as a matter of establishing without any doubt the nature of numbers as logical objects. And, since Frege thought that (HP) could not do so, it was rejected in order to give space for something that, in principle, could: the explanation of numbers in terms of extensions of concepts (value-ranges). Moreover, the above passage also points out that - at least before his acknowledgement of Russell's paradox - value-ranges had for Frege a special status as the most fundamental justification of logical objects. ${ }^{36}$ That is, logical objects are apprehended as value-ranges of functions, which, in turn, are ruled by Axiom V. This, however, is not a mere axiom for him: it is a fundamental law of logic.

Taking all of this into consideration, one should expect that the presence of urelements would play a minor role in Frege's theory, since what is at stake for him is the construction of mathematical objects on a logical basis, which is carried out through the notion of value-ranges. This view also explains his procedure of identifying truth-values with value-ranges in GGA $\S 10$, and not with other objects: since truth-values and value-ranges are both logical objects, but the latter are the most basic ones, it goes without saying that truth-values should be constructed and apprehended by means of some particular value-ranges. It is also natural to think that there is no Caesar problem for Frege after numbers are defined as value-ranges, given the undisputed nature of the latter as logical objects in his account.

This view on the Caesar problem proves its worth through its consistence with all of the aspects of GGA relevant to the absence of urelements that I have been discussing. Together with my previous considerations, it stresses why Frege claimed to have fixed the reference of value-range names and thought that this referential determination could be performed without any mention of urelements at all, resulting in the restriction of his principle of complete determination to logical objects. It can be seen, therefore, that Frege did not fail to solve a "persistent version" of the Caesar problem in GGA, simply because, according to his standards, there has never been such problem, nor any sort of indeterminacy of the notion of value-ranges left..$^{37}$

Acknowledgments I am indebted to Alessandro Duarte, Dirk Greimann, Marco Ruffino, and an anonymous referee for valuable

\footnotetext{
${ }^{36}$ For a more detailed account of this view, see Ruffino (2000, 2002).

${ }^{37}$ Insofar as the domain of the system of GGA is limited to logical objects, see
} fn. 20 . 
comments on previous drafts of this paper. Special thanks are due to Øystein Linnebo and Fernando Raul Neto for helpful discussions. I am grateful to CAPES (Brazil) for its support while work on this paper was begun.

Institute of Logic and Cognition

Department of Philosophy

Sun Yat-sen University

Guangzhou

China

b.bentzen@hotmail.com

\section{References}

Bentzen, B., "Value-ranges, Julius Caesar and Indeterminacy," M.A. Thesis, Universidade Federal de Pernambuco (2014).

Boolos, G, "The consistency of Frege's Foundations of Arithmetic," pp. 211-233 in On Being and Saying: Essays for Richard Cartwright, edited by J. J. Thomson, MIT Press, Cambridge, 1981.

Burge, T., "Frege on Extensions of Concepts, from 1884 to 1903," The Philosophical Review, vol. 93 (1984), pp. 3-34.

Dummett, M., The Interpretation of Frege's Philosophy, Duckworth, London and Harvard University Press, Cambridge, 1981.

Dummett, M., Frege: Philosophy of Mathematics, Harvard University Press, Cambridge, 1991.

Dummett, M., Origins of Analytical Philosophy, Duckworth, London and Harvard University Press, Cambridge, 1993.

Frege, G., Begriffsschrift, Louis Nebert, Halle a. S, 1879.

Frege, G., Die Grundlagen der Arithmetik, W. Koebner, Breslau, 1884. Translated by J. L. Austin as The Foundations of Arithmetic, Blackwell, Oxford, 1950.

Frege, G., Die Grundgesetze der Arithmetik (I-II), Georg Olms, Hildesheim, 1962.

Frege, G., "Letter to Russell (June 22 1902)," in pp. 127-128, From Frege to Gödel, translated by B. Woodward, edited by J. van Heijenoort, Harvard University Press, Cambridge, 1967.

Frege, G., "Letter to Russell (July 28 1902)," in pp. 152-154, Philosophical and Mathematical Correspondence, translated by $\mathrm{H}$. Kaal, edited by G. Gabriel, H. Hermes, F. Kambartel, C. Thiel, A. Veraart, Blackwell, Oxford, 1980. 
Frege, G., The Basic Laws of Arithmetic: Exposition of the System, edited and translated by M. Furth, University of California Press, Berkeley, 1964.

Frege, G., "Über die Grundlagen der Geometrie (Part I)," Jahresbericht der Deutschen Mathematiker-Vereinigung, pp. 319324, 1903, Collected Papers on Mathematics, Logic and Philosophy, translated by M. Black, V.H. Dudman, P. Geach, H. Kaal, E.-H. W. Kluge, B. McGuinness, R.H. Stoothoff and edited by B. McGuinness, Blackwell, Oxford, 1984.

Geach, P. T. and Black, M., Translations from the Philosophical Writings of Gottlob Frege, Blackweil, Oxford, 1960.

Greimann, D, "What is Frege's Julius Caesar Problem?," Dialectica, vol. 53 (2003), pp. 261-278.

Heck, R, "Julius Caesar Objection," pp. 273-308 in Language, Thought, and Logic: Essays in Honour of Michael Dummett, edited by R. Heck, Oxford University Press, New York and Oxford, 1981.

Heck, R, "Grundgesetze der Arithmetik, I, §§29-32," Notre Dame Journal of Formal Logic, vol. 38 (1997), pp. 437-474.

Heck, R, "Grundgesetze der Arithmetic I §10," Philosophia Mathematica, vol. 7 (1999), pp. 258-292.

Heck, R, "Julius Caesar and Basic Law V," Dialectica, vol. 59 (2005), pp. $161-178$.

Hintikka, J. and Sandu, G. "The skeleton in Frege's cupboard: The standard versus nonstandard distinction," The Journal of Philosophy, vol. 89 (2005), pp. 290-315.

Kemp, G., "Caesar from Frege's Perspective," Dialectica, vol. 59 (2005), pp. 179-199.

Landini, G., Frege's notations: What they are and how they mean, Palgrave Macmillan, Basingstoke, 2012.

Linnebo, Ø., "Frege's proof of referentiality," Notre Dame Journal of Formal Logic, vol. 45 (2004), pp. 73-98.

Linnebo, Ø., "To be is to be an F," Dialectica, vol. 59 (2005), pp. 201-222.

MacBride, F, "More problematic than ever: the Julius Caesar Objection," pp. 174-202 in Identity and Modality, edited by F. MacBride and C. Wright, Oxford University Press, Oxford, 2006.

Martin Jr, E., "Referentiality in Frege's Grundgesetze," History and Philosophy of Logic, vol. 3 (1982), pp. 151-164.

Moore, A. W. and Rein, A., "Grundgesetze Section 10," pp. 375- 
384 in Frege Synthesized, edited by L. Haaparanta and J. Hintikka, Springer, Dordrecht, 1986.

Moore, A. W. and Rein, A., "Frege's Permutation Argument," Notre Dame Journal of Formal Logic, vol. 28 (1987), pp. 51-54.

Parsons, C., "Frege's Theory of Number," pp. 180-203 in Philosophy in America, edited by M. Black, Allen \& Unwin, London, 1965.

Resnik, M., "Frege's proof of referentiality," pp. 177-195 in Frege Synthesized, edited by L. Haaparanta and J. Hintikka, Springer, Dordrecht, 1986.

Ricketts, T., "Truth-values and courses-of-value in Frege's Grundgesetze," pp. 177-195 in Early Analytic Philosophy: Frege, Russell, Wittgenstein, edited by W. Tait, Open Court, Chicago, 1997.

Ruffino, M., "Extensions as representative objects in Frege's logic," Erkenntnis, vol. 52 (2000), pp. 232-252.

Ruffino, M., "Logical Objects in Frege's Grundgesetze, Section 10," pp. 125-148 in From Frege to Wittgenstein, edited by E. Reck, Oxford University Press, Oxford, 2002.

Schroeder-Heister, P., "A model-theoretic reconstruction of Frege's Permutation Argument," Notre Dame Journal of Formal Logic, vol. 28 (1987), pp. 69-79.

Sluga, H., Frege, Routledge and Kegan Paul, London, 1980.

Schirn, M., "Percursos de Valores e Indeterminação da Referência," Princípios, vol. 8 (2001), pp. 36-48.

Wright, C., Frege's Conception of Numbers as Objects, Aberdeen University Press, Aberdeen, 1983. 